\title{
Hepatic pathology in patients dying of COVID-19: a series of 40 cases including clinical, histologic, and virologic data
}

\author{
Stephen M. Lagana $\mathbb{D}^{1} \cdot$ Satoru Kudose ${ }^{1} \cdot$ Alina C. Iuga $^{1} \cdot$ Michael J. Lee $^{1} \cdot$ Ladan Fazlollahi $^{1} \cdot$ Helen E. Remotti $\mathbb{D}^{1} \cdot$ \\ Armando Del Portillo ${ }^{1}$ Simona De Michele ${ }^{1}$ - Anne Koehne de Gonzalez ${ }^{1}$. Anjali Saqi ${ }^{1} \cdot$ Pascale Khairallah $^{2}$. \\ Alexander M. Chong ${ }^{3} \cdot$ Heekuk Park ${ }^{3} \cdot$ Anne-Catrin Uhlemann $^{3} \cdot$ Jay H. Lefkowitch ${ }^{1}$ Elizabeth C. Verna ${ }^{4}$
}

Received: 30 June 2020 / Revised: 30 July 2020 / Accepted: 30 July 2020 / Published online: 13 August 2020

(c) The Author(s), under exclusive licence to United States \& Canadian Academy of Pathology 2020

\begin{abstract}
The novel coronavirus SARS-CoV-2 (coronavirus disease 19, or COVID-19) primarily causes pulmonary injury, but has been implicated to cause hepatic injury, both by serum markers and histologic evaluation. The histologic pattern of injury has not been completely described. Studies quantifying viral load in the liver are lacking. Here we report the clinical and histologic findings related to the liver in 40 patients who died of complications of COVID-19. A subset of liver tissue blocks were subjected to polymerase chain reaction (PCR) for viral ribonucleic acid (RNA). Peak levels of alanine aminotransferase (ALT) and aspartate aminotransferase (AST) were elevated; median ALT peak $68 \mathrm{U} / 1$ (normal up to $46 \mathrm{U} / \mathrm{l}$ ) and median AST peak $102 \mathrm{U} / 1$ (normal up to $37 \mathrm{U} / \mathrm{l}$ ). Macrovesicular steatosis was the most common finding, involving 30 patients $(75 \%)$. Mild lobular necroinflammation and portal inflammation were present in 20 cases each (50\%). Vascular pathology, including sinusoidal microthrombi, was infrequent, seen in six cases (15\%). PCR of liver tissue was positive in 11 of 20 patients tested (55\%). In conclusion, we found patients dying of COVID-19 had biochemical evidence of hepatitis (of variable severity) and demonstrated histologic findings of macrovesicular steatosis and mild acute hepatitis (lobular necroinflammation) and mild portal inflammation. We also identified viral RNA in a sizeable subset of liver tissue samples.
\end{abstract}

\section{Introduction}

The pandemic caused by the novel coronavirus SARS-CoV2 (COVID-19) has resulted in significant pulmonary morbidity and mortality, but has also raised many questions

Supplementary information The online version of this article (https:// doi.org/10.1038/s41379-020-00649-x) contains supplementary material, which is available to authorized users.

$\triangle$ Stephen M. Lagana

sm12179@cumc.columbia.edu

1 Department of Pathology and Cell Biology, Columbia University Irving Medical Center, New York, NY, USA

2 Department of Medicine, Division of Nephrology, Columbia University Irving Medical Center, New York, NY, USA

3 Department of Medicine, Division of Infectious Diseases and Microbiome \& Pathogen Genomics Core, Columbia University Irving Medical Center, New York, NY, USA

4 Center for Liver Disease and Transplantation, Columbia University Irving Medical Center, New York, NY, USA regarding involvement of other organ systems such as the liver. Hepatologists and liver pathologists were therefore interested to find that researchers from China demonstrated liver function test (LFT) abnormalities in $44 \%$ of COVID19 patients and that these abnormalities seemed to correlate with the severity of pulmonary disease [1, 2]. Histologic reports from China were limited. A small case series reported the presence of fat in hepatocytes, with macrovesicular steatosis depicted in the accompanying photomicrograph (although described in the text as microvesicular fat) [3]. A more recent study performed core needle biopsies on the livers of four patients and reported nonspecific findings, attributed to preexisting disease or perimortem injury [4]. Our group recently described moderate acute hepatitis in a liver allograft recipient with concomitant acute cellular rejection [5, 6]. Nonetheless, the spectrum of histological liver injury in COVID-19 remains unknown. A central question has been whether the virus specifically infects the liver, or is the liver injury entirely related to the "cytokine storm" that some patients exhibit, or is a combination of these factors present. The angiotensin converting enzyme receptor 2 (ACE2) has been suggested 
to be the main mode of entry for the virus into the cell. It is found abundantly on cholangiocytes, but only rarely on hepatocytes; though it may be upregulated on hepatocytes at times of physiologic stress [7]. ACE2 is also expressed on endothelial cells, such as those found in the portal vasculature [8]. Following the SARS-CoV outbreak of 2002, virus was reported in liver and features of acute hepatitis (referred to as "bystander hepatitis") were described [9].

Early in the course of the pandemic, New York City was the global epicenter for cases of, and deaths from, COVID19. Our volume of autopsies increased sharply, and most of the cases were due to sequelae of COVID-19. The lung findings predominated, as expected; however, $2 / 3$ of COVID-19 patients in our healthcare system had abnormal LFTs [10]. Therefore, we characterized the liver findings (clinically and pathologically) in 40 autopsy cases. We also investigated whether viral ribonucleic acid (RNA) could be detected in liver tissue by polymerase chain reaction (PCR).

\section{Methods}

Following approval from the Columbia University Irving Medical Center Institutional Review Board, we reviewed lung and liver sections from 40 consecutive autopsies from patients who died of illness related to COVID-19. All liver slides were reviewed by an experienced liver pathologist (SML) and any findings (or potential findings), which are uncommonly identified in routine practice (e.g., phlebosclerosis), were reviewed amongst the liver pathology group for consensus. Sections were typical autopsy sections $(\sim 2$ $\mathrm{cm} \times 2 \mathrm{~cm}$ ) taken from representative appearing area(s), formalin fixed and paraffin embedded (FFPE). Hematoxylin and eosin $(\mathrm{H} \& \mathrm{E})$ was the only stain routinely employed, though if further stains were used for clinical purposes, they were reviewed as deemed relevant. PCR was performed on selected cases for which additional tissue blocks of liver were available. RNA from samples was extracted using Quick-RNA FFPE Miniprep (ZYMO RESEARCH). Quantitative RT-PCR was performed on extracted RNA using Taqman 4X master mix and SARS-COV-2 primer/ probe sets (IDT) to detect presence of virus per the CDC recommendations [11]. Each assay included a standard

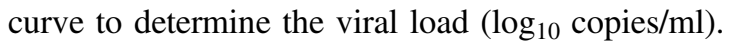

Demographic and clinical data were collected on all cases as available, specifically: age, gender, length of hospital stay, body mass index (BMI), hypertension, diabetes mellitus, chronic kidney disease, and chronic heart disease. In addition, charts were interrogated for evidence of known or suspected chronic liver disease, including viral hepatitis, alcohol-related liver disease, nonalcoholic fatty liver disease (NAFLD), autoimmune liver disease, transplantation status, and/or cirrhosis. Laboratory values were also investigated (initial and peak), including aspartate aminotransferase (AST), alanine aminotransferase (ALT), total bilirubin (TB), albumin (initial and nadir), creatinine, ferritin, Ddimer, c-reactive protein (CRP), and interleukin-6 (IL-6). Certain therapeutic interventions were investigated including administration of corticosteroids, hydroxychloroquine, and tocilizumab (IL- 6 inhibitor).

Continuous variables were expressed as median (25th-75th percentile). Categorical variables were shown as fraction (percentage). Clinical and pathologic features among those with and without detectable virus by PCR and among those with and without acute lung injury (ALI) were compared using Fisher's exact test for categorical variables and Mann-Whitney $U$ test for continuous variables. In addition, differences in the type of therapeutic intervention and length of stay stratified by the distribution and extent of steatosis and lobular or portal inflammation were assessed using Fisher's exact test or Kruskal-Wallis test as appropriate. Patients with missing data were excluded from the analysis. All statistical analyses were performed using $\mathrm{R}$ (version 3.6.1).

Lung findings were simplified for the purposes of this report into "ALI" and "no ALI." ALI comprised the histologic spectrum of diffuse alveolar damage (DAD) with an acute (exudative) phase demonstrating hyaline membranes with or without an organizing (proliferative) phase exhibiting interstitial fibroblastic proliferation as well as a single case with predominantly fibrin, compatible with acute and fibrinous pneumonia.

\section{Results}

We sequentially examined the liver sections of the first 44 COVID-19 autopsies at our institution; however, four were excluded for severe autolysis resulting in a cohort of 40 patients. The overall median (IQR) age was 70 (66-80) years and $29(70 \%)$ were men. Twenty-three patients were Hispanic, five were African American, and two were Caucasian (the remaining ten were unknown). Seven of the patients had died on arrival, and had either no or limited clinical data. The median length of stay was 8.5 days. Twenty-two patients $(55 \%)$ received steroids during their admission, $19(47.5 \%)$ received hydroxychloroquine, and six $(15 \%)$ received tocilizumab (this cohort was encountered before remdesivir was widely used, and none of these patients received it). Patient characteristics and known comorbidities are summarized in Table 1.

There were two patients with evidence of chronic liver disease, one with alcohol-related cirrhosis and one with a history of liver transplant for autoimmune-related liver disease and acute cellular rejection at the time of admission. Five patients had imaging evidence of NAFLD on 
Table 1 Summary of demographics and past medical history $(N=40)$.

Demographics

\begin{tabular}{ll}
\hline Age & $70(66,80)$ \\
Sex & \\
$\quad$ Female & $12(30 \%)$ \\
$\quad$ Male & $28(70 \%)$ \\
BMI, $N=32$ & $26.5(23.9,32.0)$ \\
Past medical history & \\
Chronic liver disease, $N=16^{\mathrm{a}}$ & $2 / 16(13 \%)$ \\
Diabetes & $18 / 36(50 \%)$ \\
Hypertension, $N=38$ & $29 / 38(76 \%)$ \\
Chronic kidney disease, $N=32$ & $7 / 32(22 \%)$ \\
Cardiac disease, $N=28$ & $10 / 28(36 \%)$ \\
\hline
\end{tabular}

All statistics are presented as median (interquartile range) or $N(\%)$.

$B M I$ body mass index.

${ }^{\text {a }}$ Chronic liver disease $N$ based on patients in whom we had preadmission data.

admission. In addition, one patient with imaging evidence of steatosis also had an isolated anti-hepatitis B (HBV) core antibody positive with low-level HBV DNA detected.

Initial and peak laboratory values including liver enzymes and inflammatory markers are displayed in Table 2. The median initial and peak AST and ALT were 1-3 times the upper limit of normal, while median TB values were in the normal range. Kidney dysfunction was common with a median peak creatinine of $2.64 \mathrm{mg} / \mathrm{dl}$ (upper limit of normal $=0.98 \mathrm{mg} / \mathrm{dl}$ for females and 1.30 $\mathrm{mg} / \mathrm{dl}$ for males). Median peak levels of inflammatory markers including CRP $(268 \mathrm{mg} / \mathrm{l}$, upper limit of normal = $10 \mathrm{mg} / \mathrm{l})$, ferritin $(1810 \mathrm{ng} / \mathrm{ml}$, upper limit of normal = $150 \mathrm{ng} / \mathrm{ml}$ for females, $400 \mathrm{ng} / \mathrm{ml}$ for males), D-dimer $(9.6 \mu \mathrm{g} / \mathrm{ml}$, upper limit of normal $=0.8 \mu \mathrm{g} / \mathrm{ml})$, and IL-6 $(>315 \mathrm{pg} / \mathrm{ml}$, upper limit of normal $=5 \mathrm{pg} / \mathrm{ml})$ were all markedly elevated. There were no significant associations between laboratory values and any specific histological feature (data not shown).

Grossly, two livers showed fibrosis and one had abscesses, the remaining livers showed varying degrees of steatosis, congestion, and ischemia, but no other significant gross pathology. Histologically, the most frequently encountered findings were macrovesicular steatosis, mild acute hepatitis, and minimal-to-mild portal inflammation. Various less frequent findings were also observed. The findings are described below and major findings are summarized in Table 3 . As the focus of this study is liver pathology, the lungs were considered only in the context of how the pulmonary findings may relate to liver injury. Overall, 29 of 40 patients (73\%) had evidence of ALI histologically (the gross appearance of the lungs was not reviewed for this study). The patients with ALI did not differ from those without with
Table 2 Summary of laboratory results.

\begin{tabular}{ll}
\hline AST $(\mathrm{U} / \mathrm{l}), N=33$ & \\
$\quad$ Initial & $63(43,92)$ \\
$\quad$ Peak & $102(54,294)$ \\
ALT $(\mathrm{U} / \mathrm{l}), N=33$ & \\
$\quad$ Initial & $32(19,55)$ \\
$\quad$ Peak & $68(32,258)$ \\
Albumin $(\mathrm{g} / \mathrm{dl}), N=33$ & \\
$\quad$ Initial & $3.50(3.10,3.90)$ \\
$\quad$ Nadir & $2.40(1.80,3.10)$ \\
TB (mg/dl), $N=33$ & \\
$\quad$ Initial & $0.50(0.40,0.90)$ \\
$\quad$ Peak & $0.90(0.60,1.50)$ \\
Creatinine $(\mathrm{mg} / \mathrm{dl}), N=34$ & \\
$\quad$ Initial & $1.40(1.08,2.29)$ \\
Peak & $2.64(1.63,4.44)$ \\
CRP (mg/l), $N=31$ & \\
$\quad$ Initial & $190(41,248)$ \\
$\quad$ Peak & $268(172,300)$ \\
Ferritin (ng/ml), $N=29$ & \\
Initial & $594(302,1635)$ \\
Peak & $1810(571,4460)$ \\
IL-6 (pg/ml), $N=25$ & \\
Initial & $96(66,248)$ \\
Peak & $315(206,315)$ \\
D-dimer $(\mu \mathrm{g} / \mathrm{ml}), N=27$ & \\
Initial & $2.8(1.2,8.4)$ \\
Peak & $9.6(5.8,20.0)$ \\
\hline All &
\end{tabular}

All statistics are presented as median (interquartile range).

$A L T$ alanine aminotransferase, AST aspartate aminotransferase, $C R P$ C-reactive protein, $T B$ total bilirubin.

Table 3 Summary of pathologic parameters $(N=40)$.

\begin{tabular}{lr}
\hline Acute lung injury (histologic) $^{\mathrm{a}}$ & $29 / 40(72 \%)$ \\
Lobular necroinflammation & $20 / 40(50 \%)$ \\
Portal inflammation & $20 / 40(50 \%)$ \\
Lobular apoptosis (individual hepatocytes) & $10 / 40(25 \%)$ \\
Steatosis (>5\%) & $30 / 40(75 \%)$ \\
Fat percent & \\
$\quad 0-5 \%$ & $10 / 40(25 \%)$ \\
$6-33 \%$ & $19 / 40(48 \%)$ \\
$34-66 \%$ & $6 / 40(15 \%)$ \\
$\quad 67-100 \%$ & $5 / 40(12 \%)$ \\
Cholestasis & $15 / 40(38 \%)$
\end{tabular}

All statistics are presented as $N(\%)$.

${ }^{a}$ Acute lung injury includes diffuse alveolar damage including exudative and proliferative phases and single case of acute fibrinous and organizing pneumonia.

respect to any of the laboratory variables we cataloged, or to any histologic findings (data not shown). There was, however, a trend toward increased serum CRP in the ALI group $(282 \mathrm{mg} / \mathrm{l}$ vs. $218 \mathrm{mg} / \mathrm{l}, p=0.07)$. 


\section{Steatosis}

Steatosis was common, effecting 30 patients (75\%), Fig. 1a. Fat droplets were predominantly macrovesicular and no case of true microvesicular steatosis was encountered. Two cases $(7 \%)$ had active steatohepatitis with ballooning and Mallory-Denk bodies. Nineteen patients (48\%) had mild fat (6-33\%), six patients (15\%) had moderate fat (34-66\%), and five patients $(12 \%)$ had marked fat $(>67 \%)$. Most commonly, the fat was panlobular (zones 1, 2, and 3; periportal to centrilobular, 18 cases, 53\%). The fat was restricted to the centrilobular region (zone 3 ) in seven cases $(21 \%)$, while it was located in zones 2 and 3 in three cases $(8.8 \%)$. Ten $(33 \%)$ cases had "mismatched" fat, in which the steatosis was mild, but the fat involved periportal (zone 1) hepatocytes. A BMI was calculable in 32 patients. Two of 32 had a BMI over 35. There was no correlation between BMI and percent steatosis (Spearman's $r=-0.04, p=$ $0.84)$. Similarly, there was no association between length of stay and percent steatosis $(p=0.57)$ or distribution (zonality) of steatosis $(p=0.80)$. Half of the patients in whom we had data (18 of 36) were diabetic. Having a history of diabetes mellitus was not associated with percent steatosis $(p=0.48)$ or distribution of steatosis $(p=0.43)$. Treatment with corticosteroids was not associated with percent steatosis $(p=0.35)$ or distribution of steatosis $(p=0.87)$.

\section{Acute hepatitis}

A total of 20 cases (50\%) showed features of acute hepatitis, defined as the presence of lobular necroinflammation, Fig. 1b. These foci contained lymphocytes and rare histiocytes. Plasma cells were rare. Sixteen of these cases were of mild severity (80\%) and four were of moderate severity (20\%). No severe hepatitis (e.g., submassive necrosis, massive hepatic necrosis, bridging necrosis) was encountered. Four patients without necroinflammation showed rare, individual apoptotic hepatocytes. Given the focality of the finding (one or two such cells in one or two sections of liver), the significance of this observation is uncertain. Six cases with lobular necroinflammation also demonstrated occasional individual apoptotic hepatocytes, Fig. 1c. Lobular mitoses were seen in three cases (8\%). Two of these cases also had acute hepatitis, whereas one occurred in an allograft which did not demonstrate active hepatitis. There were no associations between lobular necroinflammation and length of stay $(p=0.87)$, hydroxychloroquine treatment $(p=0.73)$, or tocilizumab administration $(p=0.17)$.

\section{Portal inflammation}

Twenty patients (50\%) had portal inflammation. Three cases had interface hepatitis (equivalent to Batts-Ludwig Grade 2, Fig. 1d), whereas 17 had only minimally increased portal mononuclear cells (lymphocytes and few portal macrophages). One of the cases with interface occurred in an allograft with recent severe rejection with autoimmune features (ACR-AIH). Eosinophils and neutrophils were rare, and not prominent in any case. An occasional plasma cell was present, but in none of the cases were they significant enough to invoke consideration of autoimmune hepatitis or drug induced liver injury with autoimmune features (DILI-AIH), with the exception of the ACR-AIH case. No case demonstrated
Fig. 1 Common autopsy findings. a An example of marked steatosis involving all three zones $(\times 100)$. This patient had a body mass index of 25 . b A typical focus of lobular necroinflammation, comprised predominantly of lymphocytes with admixed apoptotic debris ( $\times 600)$. c A single apoptotic hepatocyte (arrow, x600). Panel $\mathbf{d}$ is taken from one of the rare cases with interface hepatitis $(\times 200)$. All images depict hematoxylin and eosin stained slides.

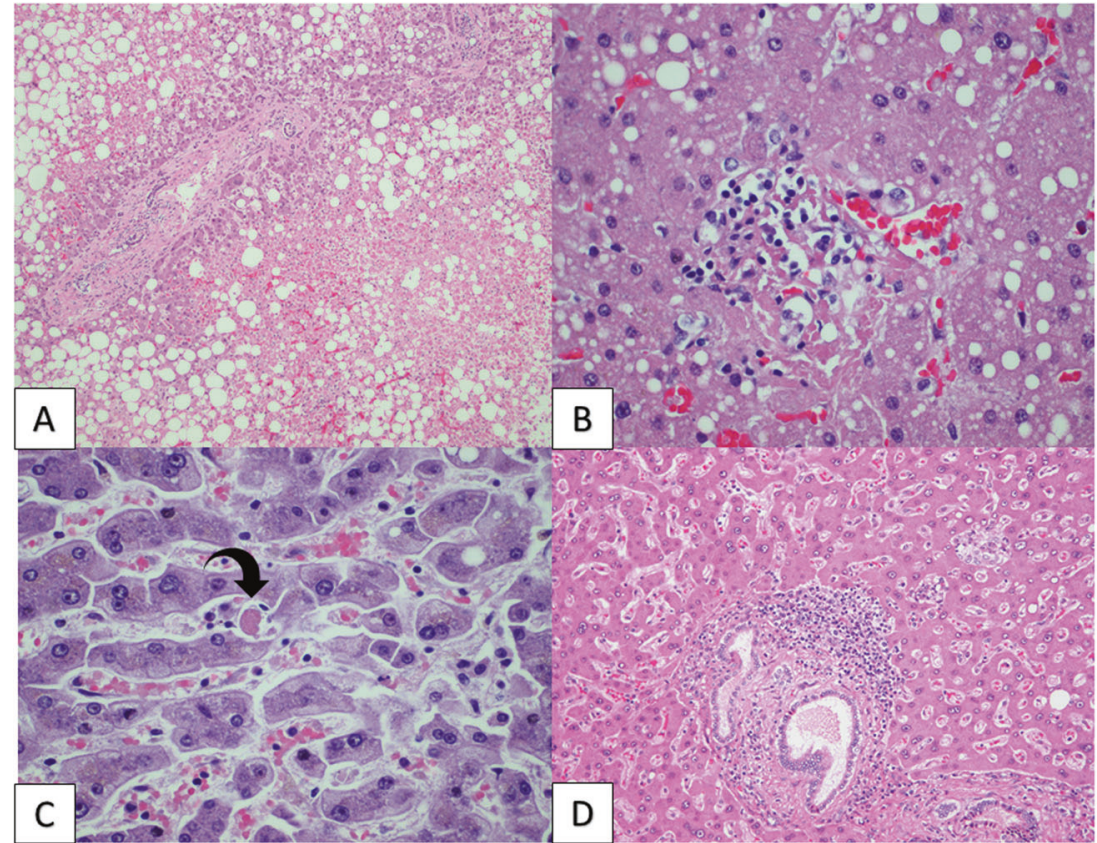


lymphoid aggregates. Overall, 6 of the 20 patients did not have evidence of lobular/acute hepatitis. Of these one was the ACR-AIH patient and two came into the emergency room in cardiopulmonary arrest, and without history in our system. There were no associations between portal inflammation and length of stay $(p=0.86)$, hydroxychloroquine treatment $(p=$ $0.20)$, or tocilizumab administration $(p=0.10)$.

\section{Perimortem pathology}

In cases with acute, severe cardiac dysfunction areas of congestion (right heart failure) and ischemia (left heart failure) are common regardless of etiology. In our series, 32 (78\%) patients exhibited congestion and $16(40 \%)$ exhibited centrilobular ischemic necrosis.

\section{Biliary findings}

Fifteen (38\%) cases had lobular cholestasis, generally mild and focal. Four of these 15 cases (10\% of series) had ductular cholestasis, indicative of sepsis. Interlobular bile ducts were present in a normal distribution in all cases (no ductopenia) and the ducts were normal appearing (tubular structures composed of cholangiocytes with maintained polarity) in 39 of 40 cases (the ACR-AIH case had duct dysmorphia).

\section{Vascular pathology}

None of the cases had diffuse vascular pathology; however, focal findings were identified. Phlebosclerosis, reminiscent of veno-occlusive disease (VOD) was present in six cases, Fig. 2a. In five cases, this occurred in a portal venule, but one case had involvement of the central vein. Portal arterioles were abnormal in nine cases. Three of these had arteriolar muscular hyperplasia, Fig. 2b. Each case with muscular hypertrophy of the portal arterioles also had venous phlebosclerosis. Four cases had hyalinosis of the vessel wall (Fig. 2b). Two cases had fibrinoid necrosis with endothelial apoptosis (Fig. 2c). Sinusoidal microthrombi were present in six cases (Fig. 2d). C4d immunohistochemistry (IHC) was performed on ten cases and was negative (either entirely or almost entirely negative in all cases).

\section{Granulomas}

Three cases had granulomatous inflammation. One case had portal and lobular granulomas reminiscent of "fibrin ring" morphology, Fig. 3a. This patient had been treated with a number of potentially hepatotoxic medications throughout the hospital course including hydroxychloroquine and tocilizumab for severe COVID-19, as well as amiodarone for atrial fibrillation and ceftriaxone and piperacillin/tazobactam for infection. One patient had multiple necrotizing granulomas forming grossly appreciable abscesses and had structures suggestive of Schistosoma eggs. The final case demonstrated portal nonnecrotizing granulomas in two portal tracts and resembled primary biliary cholangitis (PBC). However, the patient was male, had no known history of PBC, and had a normal alkaline phosphatase.
Fig. 2 Vascular findings. a Phlebosclerosis (arrow) involving a portal venule. b Portal arteriolar muscular hyperplasia (lower arrow), a finding which was only observed in cases with phlebosclerosis. The upper arrow highlights hyalinosis of a smaller branch of portal arteriole. c A portal tract in which a small branch of portal venule had apoptotic endothelial cells and focal fibrinoid necrosis (arrow). d A sinusoidal thrombus. All images depict hematoxylin and eosin stained slides magnified $\times 600$.

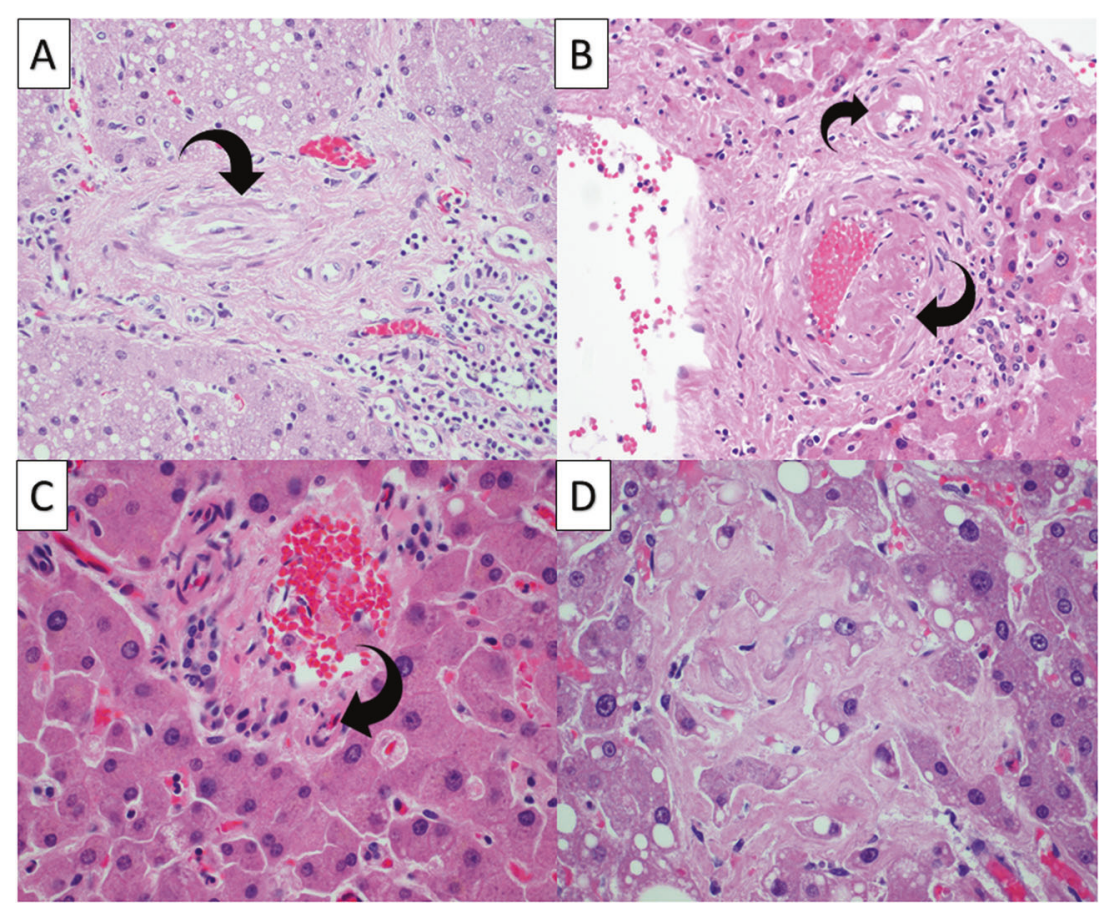




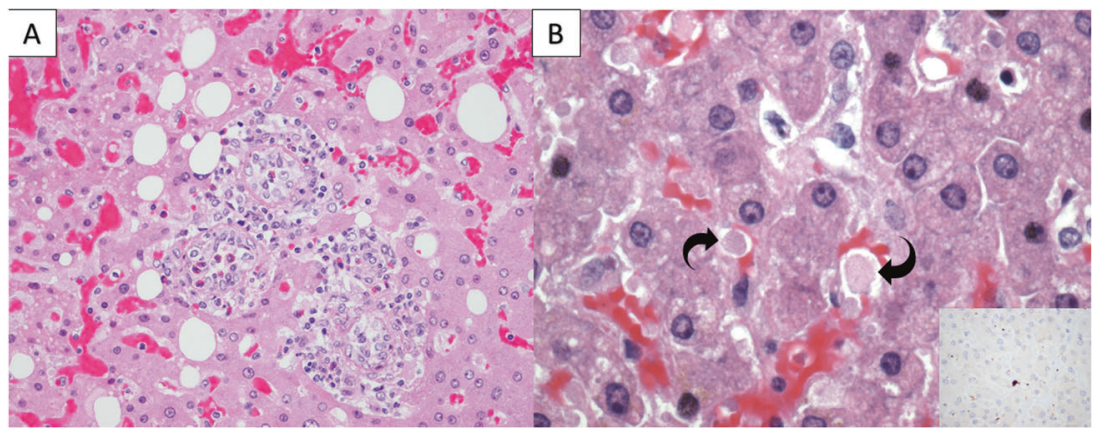

Fig. 3 Uncommon and unusual findings. a Prominent lobular granulomas resembling fibrin ring granulomas that occurred in a patient on multiple medications $(\times 400)$. Panel $\mathbf{b}$ demonstrates "thrombotic bodies" (arrows), while the inset demonstrates CD61 positivity in the

\section{Thrombotic bodies}

Two cases had pale ovoid sinusoidal inclusions, which at low power resembled apoptotic hepatocytes. Upon close examination, it became evident that these inclusions were present in the sinusoidal spaces, Fig. 3b. We stained these with CD61, which was positive (Fig. 3b inset), supporting that these aggregates were rich in platelets. We chose to refer to these structures as "thrombotic bodies."

\section{Viral PCR}

PCR was performed on 20 autopsy livers and was positive in $11(55 \%)$ ranging from 10 copies to 9254 copies/ $\mu$ RNA. The longest interval between initial diagnosis by nasopharyngeal swab and death was 25 days and the median was 13 days. There was no relationship between time from initial diagnosis (positive nasopharyngeal swab) and likelihood of PCR positivity in the liver at autopsy $(p=0.51)$. We investigated whether PCR positivity was associated with any laboratory parameters (Table 4). Median peak AST was higher among patients with a positive PCR compared to negative ( 239 vs. $86 \mathrm{U} / 1$, respectively) though this did not reach statistical significance $(p=0.063)$. In addition, peak ferritin ( 3623 vs. $1014 \mathrm{ng} / \mathrm{ml}, p=0.048$ ) and peak creatinine ( 4.50 vs. $2.02 \mathrm{mg} / \mathrm{dl}, p=0.025)$ were significantly higher among PCR positive patients. There were no significant correlations between PCR positivity and any histologic finding including congestion (Supplementary Table 1).

\section{Discussion}

Here, we present the hepatic findings in 40 autopsies of patients who died of complications of COVID-19. Excluding perimortem changes (congestion and ischemia) the main findings were hepatic steatosis $(75 \%)$, mild acute hepatitis (50\%), and portal inflammation (50\%). We structures, suggesting they are comprised largely of platelets $(\times 600)$. Main panels depict hematoxylin and eosin stained slides, whereas inset is anti-CD61 $(\times 600)$.

Table 4 Laboratory results among stratified by PCR positivity $(N=20)$.

\begin{tabular}{|c|c|c|c|}
\hline $\begin{array}{l}\text { Laboratory } \\
\text { results }\end{array}$ & $\begin{array}{l}\text { PCR negative } \\
(N=9)\end{array}$ & $\begin{array}{l}\text { PCR positive } \\
(N=11)\end{array}$ & $p$ value* \\
\hline \multicolumn{4}{|c|}{ AST (U/l), $N=20$} \\
\hline Initial & $63(41,67)$ & $71(56,110)$ & 0.14 \\
\hline Peak & $86(44,294)$ & $239(96,1660)$ & 0.063 \\
\hline \multicolumn{4}{|c|}{$\operatorname{ALT}(\mathrm{U} / \mathrm{l}), N=20$} \\
\hline Initial & $37(32,53)$ & $41(22,62)$ & 0.6 \\
\hline Peak & $69(50,159)$ & $258(58,440)$ & 0.3 \\
\hline \multicolumn{4}{|c|}{ Albumin $(\mathrm{g} / \mathrm{dl}), N=20$} \\
\hline Initial & $3.60(3.20,3.90)$ & $3.20(2.85,3.75)$ & 0.5 \\
\hline Nadir & $2.20(1.90,2.90)$ & $1.80(1.65,2.25)$ & 0.2 \\
\hline \multicolumn{4}{|c|}{$\mathrm{TB}(\mathrm{mg} / \mathrm{dl}), N=20$} \\
\hline Initial & $0.50(0.40,0.50)$ & $0.70(0.45,1.45)$ & 0.14 \\
\hline Peak & $1.0(0.6,1.2)$ & $2.2(0.7,2.8)$ & 0.15 \\
\hline \multicolumn{4}{|c|}{ Creatinine $(\mathrm{mg} / \mathrm{dl}), N=20$} \\
\hline Initial & $1.31(1.04,1.44)$ & $1.80(1.12,2.27)$ & 0.4 \\
\hline Peak & $2.02(1.72,2.56)$ & $4.50(3.04,5.62)$ & 0.025 \\
\hline \multicolumn{4}{|c|}{$\mathrm{CRP}(\mathrm{mg} / \mathrm{l}), N=20$} \\
\hline Initial & $198(89,231)$ & $218(111,284)$ & 0.6 \\
\hline Peak & $278(248,300)$ & $285(148,300)$ & $>0.9$ \\
\hline \multicolumn{4}{|c|}{ Ferritin $(\mathrm{ng} / \mathrm{ml}), N=19$} \\
\hline Initial & $590(300,698)$ & $933(395,1954)$ & 0.3 \\
\hline Peak & $1014(702,1802)$ & $3623(1586,9948)$ & 0.048 \\
\hline \multicolumn{4}{|c|}{ D-dimer $(\mu \mathrm{g} / \mathrm{ml}), N=18$} \\
\hline Initial & $0.9(0.6,5.4)$ & $2.6(1.6,14.8)$ & 0.2 \\
\hline Peak & $8.0(5.9,20.0)$ & $20.0(14.4,20.0)$ & 0.2 \\
\hline
\end{tabular}

All statistics are presented as median (interquartile range).

$A L T$ alanine aminotransferase, $A S T$ aspartate aminotransferase, $C R P$ $C$-reactive protein, $T B$ total bilirubin.

*Wilcoxon rank-sum tests were performed.

determined that $55 \%$ of patients who died of COVID-19 had virus in the liver detected by PCR, albeit the majority at very low levels. 
Macrovesicular steatosis was common (75\%) of patients. This is consistent with other studies, for example, a study performed by a working group affiliated with the Centers for Disease Control found steatosis in $50 \%$ of autopsy livers (4/8) [12]. A high proportion of our patients were diabetics, and that could account for some of the steatosis we encountered. However, the distribution of fat in our series was not typical of NAFLD. Ten cases had only mild steatosis, but had appreciable periportal fat. Only 2 of 32 patients in whom BMI was recorded had a BMI $>35$ (median 26.5). A total of 2 of 40 patients had ballooning and Mallory-Denk bodies, indicative of alcoholic or nonalcoholic steatohepatitis. Ischemia, viral hepatitis (e.g., hepatitis $\mathrm{B}$, hepatitis $\mathrm{C}$, human immunodeficiency virus), medications (corticosteroids), malnutrition, and various other acute insults are known to cause fatty liver [13]. Steroid administration was frequent in our cohort, and this could lead to steatosis, though no direct association was seen in this cohort [14]. The facts that steatosis was not correlated to BMI or diabetes, and that there were many cases of "mismatched fat" are surprising if preexisting NAFLD explains the entirety of the histology. We did not have alcohol intake history for every patient, however alcoholic steatosis often demonstrates features of steatohepatitis with ballooning, neutrophilic satellitosis, and Mallory-Denk bodies. Features of frank steatohepatitis were present in only two cases. Therefore, it would seem likely that the steatosis in some of these cases developed during the course of their COVID-19 illness. The etiology may be multifactorial with corticosteroid administration, hypoxia, malnutrition, and direct viral effects all plausible considerations (though neither corticosteroid use nor length of stay, a surrogate for malnutrition, was statistically associated with amount or distribution of steatosis). A series of over 300 COVID-19 cases in China investigated the relationship between steatosis and neutrophil to lymphocyte ratio (NLR). A higher ratio suggests more severe systemic inflammation. This study showed that steatosis and NLR were associated with severity of illness, and also that the effects of steatosis and high NLR compounded to predict the most severe cases [15]. The exact mechanism/pathobiology of hepatic steatosis in COVID-19 is worthy of further investigation.

Mild acute hepatitis, defined as lobular necroinflammation, was present in $50 \%$ of cases. This climbs to $60 \%$ if cases with no inflammation, but with focal apoptotic hepatocytes are included. In general, individual apoptotic hepatocytes are considered as evidence of acute hepatitis, but in this series, such cases showed such infrequent hepatocyte apoptosis that the significance is uncertain. The degree of necroinflammatory activity was generally mild, but was moderate in four cases. The necroinflammatory foci consisted of one to several dead and dying hepatocytes with few accompanying lymphocytes and histiocytes. Plasma cells were only rarely encountered. The cohort of histologically defined acute hepatitis cases did not demonstrate higher serum AST/ALT levels than the cohort without lobular necroinflammation. Indeed, laboratory values were essentially identical in the histologic acute hepatitis and nonhepatitis groups. There was, however, a trend toward significance when we queried if those with PCR positivity were more likely to have evidence of acute hepatitis (44\% of PCR negative patients vs. $64 \%$ of PCR positive patients $\mathrm{did} ; p=0.08$ ). It will be interesting to investigate this variable in a larger number of cases. We have previously reported moderate acute hepatitis in a liver allograft recipient (distinct from the allograft recipient in this report) whose biopsy demonstrated large clusters of apoptotic hepatocytes with abundant lymphohistiocytic inflammation $[5,6]$. None of the cases in this series were as profoundly affected as that case. The role of immunosuppression and allograft recipient status on liver injury associated with COVID-19 will need further study.

Portal inflammation was generally minimal and was not suggestive of any specific etiology. This finding is similar to what was reported in a series of 12 autopsies in Europe, which described "minimal lymphoplasmacellular portal infiltrate" [16]. Approximately $70 \%$ of patients in our series with portal inflammation had mild acute (lobular) hepatitis. The few patients with portal inflammation, but without acute hepatitis, were a heterogenous group including an allograft recipient and several patients who arrested on arrival in the emergency room without available medical records, so it is difficult to interpret the significance of isolated portal inflammation. There was no evident bile duct injury, with the exception of the allograft with recent history of severe rejection. ACE2 receptor is present on cholangiocytes, so we were certainly interested in identifying bile duct injury, but it was not observed [17]. Cholestasis, however, was common (38\%). Four patients had ductular cholestasis, suggesting sepsis. Lobular cholestasis without injury to the bile ducts is suggestive of a hepatocellular insult; e.g., at the bile canaliculus. It is, however, possible that the cholangiocytes are functionally impaired, but that the injury is not evident by H\&E morphology. Further investigation by electron microscopy or genomic/proteomic methods may prove enlightening.

The potential role of endothelial injury in COVID-19 continues to evolve. Virus has been demonstrated in endothelial cells, and one paper reported lymphocytic endotheliitis in liver [8, 17]. In our series, however, few vascular abnormalities were histologically identified. A minority of our cases had a vascular abnormality (portal arteriole hyperplasia, VOD-like phlebosclerosis, sinusoidal thrombi), and these changes were very focal and sparse in any one histologic section. In our patient cohort, therefore, 
vascular injury did not appear to be a major form of injury. While we cannot exclude these findings being meaningful (if they are present throughout the entire liver), we do not believe that vascular injury is the major mechanism of injury in the liver in COVID-19 patients. This contrasts with a case series from Italy, which describes vascular pathology not frequently encountered in our cohort [18]. The "thrombotic bodies" we observed are likely the same "microthrombi" identified in multiple organs in a recent series of seven autopsies [19].

This study includes the largest cohort of liver tissue subjected to PCR. The results are interesting for several reasons. At the most superficial level, it is valuable to know that slightly more than half of patients had detectable virus in liver. PCR positivity was not significantly associated with AST/ALT elevations, though there was a trend for association between PCR positivity and higher peak AST (86 U/ 1 vs. $239 \mathrm{U} / 1, p=0.063$ ). As additional cases are subjected to PCR, further clarity may be obtained. There was a statistically significant elevation of ferritin and trends toward increased D-dimer and albumin nadir in the positive group. There was also statistically significantly increase in serum creatinine in the PCR positive cohort. The overall impression is that the PCR positive group had a slightly more severe inflammatory state. A detail worthy of further exploration is the variation of PCR positivity in liver. Nine cases had low viral loads (and the possibility of serum positivity cannot be excluded), whereas two cases had high viral loads. Time between initial diagnosis and death was not a factor associated with PCR positivity $(p=0.51)$. The peak creatinine elevation in the PCR positive group (4.50 vs. $2.02 ; p=0.025$ ) is a noteworthy finding, as it raises the question of whether some patients have a more disseminated phenotype and others perhaps a more anatomically (airway) restricted one. Further studies will be useful to determine if this is true, and if so, if it is clinically relevant.

This study has weaknesses, including that being an autopsy study, this paper describes patients with severe disease. As the epidemic evolves, it will be important to gather data on liver injury in patients with non-lethal COVID-19. We did not investigate pathogenesis on the cellular level. We attempted to validate in-situ hybridization (ISH) protocols and IHC for SARS-CoV-2, but nonspecific marking of lipofuscin, and reaction with endogenous alkaline phosphatase in liver tissue from non-COVID-19 patients who died years ago was nearly ubiquitous (sometimes strong and diffuse). Thus, for technical reasons, we cannot report these tissue preserving methods at present. In our clinical experience, as well as in the published literature, both ISH and IHC can be used to detect virus in lung, particularly during the acute phase of DAD, and occasionally in other organs, such as placenta [20, 21]. It will be important and interesting to attempt to identify virus in specific cells in the liver and to determine whether the ACE2 receptor is upregulated in hepatocytes in patients with severe COVID-19. Nonetheless, based on the pattern of injury observed and the results of the PCR analysis, SARS-CoV-2 seems to involve the liver, and is associated with, possibly causal of, macrovesicular steatosis and acute hepatitis.

Here, we have provided a description of the histologic, clinical, and virologic characteristics related to the liver in patients who died of sequelae of COVID-19. We have shown that patients dying of complications of COVID-19 often have abnormal liver enzymes, steatosis, mild acute hepatitis, and viral RNA in liver. While more work is needed to further elucidate the possibly multifactorial mechanisms of liver injury in patients with severe COVID19 , given the lack of association between steatosis and known NAFLD risk factors, and between hepatitic findings and known drug administration, these data suggest virally mediated liver injury based on clinical and histologic observations.

\section{Compliance with ethical standards}

Conflict of interest The authors declare that they have no conflict of interest.

Publisher's note Springer Nature remains neutral with regard to jurisdictional claims in published maps and institutional affiliations.

\section{References}

1. Chen N, Zhou M, Dong X, Qu J, Gong F, Han Y, et al. Epidemiological and clinical characteristics of 99 cases of 2019 novel coronavirus pneumonia in Wuhan, China: a descriptive study. Lancet. 2020;395:507-13.

2. Wang Q, Zhao H, Liu LG, Wang YB, Zhang T, Li MH, et al. Pattern of liver injury in adult patients with COVID-19: a retrospective analysis of 105 patients. Mil Med Res. 2020;7:28.

3. Xu Z, Shi L, Wang Y, Zhang J, Huang L, Zhang C, et al. Pathological findings of COVID-19 associated with acute respiratory distress syndrome. Lancet Respir Med. 2020;8:420-2.

4. Tian S, Xiong Y, Liu H, Niu L, Guo J, Liao M, et al. Pathological study of the 2019 novel coronavirus disease (COVID-19) through postmortem core biopsies. Mod Pathol. 2020;33:1007-14.

5. Lagana SM, De Michele S, Lee MJ, Emond JC, Griesemer AD, Tulin-Silver SA, et al. COVID-19 associated hepatitis complicating recent living donor liver transplantation. Arch Pathol Lab Med. 2020;144:929-32.

6. Heinz N, Griesemer A, Kinney J, Vittorio J, Lagana SM, Goldner $\mathrm{D}$, et al. A case of an infant with SARS-CoV-2 hepatitis early after liver transplantation. Pediatr Transplant. 2020:e13778. https://doi. org/10.1111/petr.13778.

7. Portincasa P, Krawczyk M, Machill A, Lammert F, Di Ciaula A. Hepatic consequences of COVID-19 infection. Lapping or biting? Eur J Intern Med. 2020;77:18-24.

8. Varga Z, Flammer AJ, Steiger P, Haberecker M, Andermatt R, Zinkernagel AS, et al. Endothelial cell infection and endotheliitis in COVID-19. Lancet. 2020;395:1417-8. 
9. Chau T-N, Lee K-C, Yao H, Tsang T-Y, Chow T-C, Yeung Y-C, et al. SARS-associated viral hepatitis caused by a novel coronavirus: report of three cases. Hepatology. 2004;39:302-10.

10. Phipps MM, Barraza LH, LaSota ED, Sobieszczyk ME, Pereira MR, Zheng EX, et al. Acute liver injury in COVID-19: prevalence and association with clinical outcomes in a large US cohort. Hepatology. 2020. https://doi.org/10.1002/hep.31404.

11. CDC 2019-novel coronavirus (2019-nCoV) real-time RT-PCR diagnostic panel. CDC-006-00019, CDC/DDID/NCIRD/Division of Viral Diseases; Revision: 04. Effective: 6/12/2020.

12. Roosecelis BM, Jana MR, Eduard M, Joy G, Brigid CB, Hannah $\mathrm{B}$, et al. Pathology and pathogenesis of SARS-CoV-2 associated with fatal coronavirus disease, United States. Emerg Infect Dis. 2020;26. [Epub ahead of print].

13. Milroy CM. Fatty liver and the forensic pathologist. Acad Forensic Pathol. 2018;8:296-310.

14. Ayyar VS, Almon RR, DuBois DC, Sukumaran S, Qu J, Jusko WJ. Functional proteomic analysis of corticosteroid pharmacodynamics in rat liver: relationship to hepatic stress, signaling, energy regulation, and drug metabolism. J Proteom. 2017;160:84-105.

15. Targher G, Mantovani A, Byrne CD, Wang XB, Yan HD, Sun QF, et al. Detrimental effects of metabolic dysfunction-associated fatty liver disease and increased neutrophil-to-lymphocyte ratio on severity of COVID-19. Diabetes Metab. 2020;S1262-3636:30088-4.
16. Schaller T, Hirschbühl K, Burkhardt K, Braun G, Trepel M, Märkl $\mathrm{B}$, et al. Postmortem examination of patients with COVID-19. JAMA 2020;323:2518-20.

17. Hamming I, Timens W, Bulthuis ML, Lely AT, Navis G, van Goor $\mathrm{H}$. Tissue distribution of ACE2 protein, the functional receptor for SARS coronavirus. A first step in understanding SARS pathogenesis. J Pathol. 2004;203:631-7.

18. Sonzogni A, Previtali G, Seghezzi M, Grazia Alessio M, Gianatti A, Licini L, et al. Liver histopathology in severe COVID 19 respiratory failure is suggestive of vascular alterations. Liver Int. 2020. https://doi.org/10.1111/liv.14601.

19. Rapkiewicz AV, Mai X, Carsons SE, Pittaluga S, Kleiner DE, Berger JS, et al. Megakaryocytes and platelet-fibrin thrombi characterize multi-organ thrombosis at autopsy in COVID-19: a case series. EClinicalMedicine. 2020;24:100434. [Epub ahead of print].

20. Schaefer I-M, Padera RF, Solomon IH, Kanjilal S, Hammer MM, Hornick JL, et al. In situ detection of SARS-CoV-2 in lungs and airways of patients with COVID-19. Mod Pathol. 2020. https:// doi.org/10.1038/s41379-020-0595-z.

21. Rocha AB, Stroberg E, Barton LM, Duval EJ, Mukhopadhyay S, Yarid N, et al. Detection of SARS-CoV-2 in formalin-fixed paraffin-embedded tissue sections using commercially available reagents. Lab Investig. 2020;1-5. 